\title{
Beyond lesion-load: Tractometry-based metrics for characterizing white matter lesions within fibre pathways
}

\author{
Maxime Chamberland ${ }^{1}$, Mia Winter ${ }^{1,2}$, Thomas A. W. Brice ${ }^{3}$, Derek K. \\ Jones $^{1}$, and Emma C. Tallantyre ${ }^{3,4}$ \\ 1 Cardiff University Brain Research Imaging Centre (CUBRIC), School of \\ Psychology, Cardiff University, Cardiff, United Kingdom \\ 2 Department of Clinical Neuropsychology, University Hospital of Wales, Cardiff, \\ United Kingdom \\ 3 Division of Psychological Medicine and Clinical Neurosciences, Cardiff University \\ School of Medicine, Cardiff, United Kingdom \\ 4 Helen Durham Centre for Neuroinflammation, University Hospital of Wales, \\ Cardiff, United Kingdom
}

\begin{abstract}
In multiple sclerosis studies, lesion volume (or lesion load) derived from conventional T2 imaging correlates modestly with clinical assessment. Determining which specific white matter pathways are impacted by lesions may provide additional insights regarding task-specific clinical impairment. Using diffusion MRI, we introduce a set of tractbased metrics that go beyond traditional lesion load approaches and show how they relate to task performance (i.e., working memory, information processing and verbal fluency) in a cohort of 40 patients with multiple sclerosis.
\end{abstract}

Keywords: Diffusion MRI, Tractography, Tractometry, Apparent Fiber Density, Lesion Load, Multiple Sclerosis

\section{Introduction}

Lesion load (LL) is a volumetric index derived from structural MRI often used in clinical practice to characterise the degree of damage in the brains of patients with multiple sclerosis (MS). Focal lesions on T2-weighted brain imaging in MS reflect the permanent footprint of previous episodes of inflammation [1]. Although widely used in the diagnosis, prognosis and monitoring of people with MS [2], LL correlates only modestly with clinical measures of disability; resulting in the so-called clinical-radiological paradox $[3,4]$. There are likely to be several explanations, including the occurrence of focal lesions in critical vs non-critical white matter (WM) pathways for a given cognitive function, and the presence of diffuse WM micro-structural damage that is not easily seen on conventional MRI. While LL is a convenient outcome measure in clinical studies of MS, there is a need for more advanced, and anatomically- and microstructurally-specific 
imaging metrics to allow clinicians and neuroscientists to disentangle the relationship between focal and diffuse pathology with clinical disability in MS [5].

A variety of MRI-based approaches that probe various physical properties of brain tissue have been shown to be sensitive to demyelination and axonal loss in MS [6,7] (for review, see [8]). Diffusion MRI (dMRI) allows information about the structural architecture and tissue micro-structure to be obtained by probing the random motion of water molecules [9]. The ability to derive quantitative features such as fractional anisotropy (FA) or mean diffusivity (MD) from diffusion tensor imaging (DTI) [10] and to virtually reconstruct pathways with tractography [11] has led to an exponential growth of dMRI clinico-research studies. In MS, multiple groups have examined the relationship of DTI measures within the normal-appearing WM and cognitive function [12-15]. Most studies report significant associations, although these were not always stronger than the relationship between cognitive performance and LL [3,4], potentially due to inconsistencies and limitations in tractography and associated microstructural metrics at the time.

Indeed, applying tractography to MS data can be problematic due to premature termination of streamlines within lesions $[16,17]$. However, recent advances in local modeling and tractography such as multi-tissue multi-shell constrained spherical deconvolution (MSMT-CSD, [18]) and anatomically-based tractography $[19,20]$ allow the propagation of streamlines through lesions more reliably [21-27]. Furthermore, although tractography still faces significant challenges in the field in general [28-30], recent machine learning based approaches have shown promise in reproducible tract segmentation across subjects [31]. Based on these recent methodological breakthroughs, we propose a set of tract-based metrics to improve the link between WM lesions and clinical correlates. We demonstrate the utility of the proposed metrics in a cohort of 40 MS patients.

\section{$2 \quad$ Theory and Methods}

\subsection{Clinical assessment}

40 subjects (27 women, mean age: 58 years, range: 44-78) with longstanding relapse-onset MS were recruited to this study (mean disease duration: 27 years, range: 15-47; median Expanded Disability Status Scale (EDSS) at clinical assessment: 2.5, range 0-6.0). The participants were cognitively assessed (see [32]) and the MS functional composite (MSFC, [33]) score was derived as a composite of walking speed, dexterity and information processing. Additionally, performance was assessed on three tasks involving 1) working memory (Letter-Number Sequencing, LNS [34]); 2) information processing speed (Speed of Information Processing adjusted for motor speed, SoIP [35]); and 3) verbal fluency (category switching, CF-Switching [36]). Performance on these type of tasks has been associated with compromise of widespread brain networks including fronto-temporo- 
parietal regions. This study was approved by the local ethics committee and all participants gave written informed consent.

\subsection{Acquisition}

Patients were scanned using dMRI within 12 months of their clinical assessment using a Siemens PRISMA 3T system using a 32-channel receive-only RF head coil. All participants underwent the following sequences: (1) 3D T2-weighted spin echo sequence (TR/TE: $3200 / 1408 \mathrm{~ms}$; voxel size: $\left.1 \times 1 \times 1 \mathrm{~mm}^{3}\right),(2) 3 \mathrm{D}$ T2FLAIR sequence (TR/TE: $5000 / 388 \mathrm{~ms}$, TI: $1800 \mathrm{~ms}$, voxel size $=0.5 \times 0.5 \times 0.5$ $\mathrm{mm}^{3}$ ), (3) 3D T1 MPRAGE (TR/TE: $2300 / 3 \mathrm{~ms}$; flip angle: $9^{\circ}$; voxel size: $\left.1.0 \times 1.0 \times 1.0 \mathrm{~mm}^{3}\right)$, (4) Diffusion-weighted spin-echo EPI with 14 b0 images, 30 directions at $\mathrm{b}=1200 \mathrm{~s} / \mathrm{mm}^{2}, 60$ directions at $\mathrm{b}=2400 \mathrm{~s} / \mathrm{mm}^{2}$ and $2 \times 2 \times 2 \mathrm{~mm}^{3}$ voxels.

\subsection{Processing}

Diffusion data were denoised [37] and corrected for subject motion and distortion $[38,39]$. Next, apparent fibre density (AFD) maps were derived from fiber orientation distribution functions (fODFs) obtained from MSMT-CSD [18] using a single group response function. WM lesion masks were semi-automatically delineated using 3D T2 and FLAIR images by a trained technician (co-author $\mathrm{TB}$, blinded to the purpose of the study) using NeuROI ${ }^{5}$.

For each dataset, automated WM tract segmentation was performed using TractSeg [31] to obtain the following task-relevant bundles of interest (identified by co-author MW): genu and splenium of the corpus callosum, cingulum (CG), inferior longitudinal fasciculus (ILF) and uncinate fasciculus (UF). For each bundle, 2000 streamlines were generated. The AFD was then averaged within each bundle of interest. A whole brain set of streamlines was also derived by concatenating all TractSeg outputs in each subject.

\subsection{Proposed metrics}

LL (Fig. 1a) is typically defined as the total volume $\left(\mathrm{mm}^{3}\right)$ of the lesions $\left(V_{\text {les }}\right)$, or its normalized variation:

$$
\mathrm{LL}=\frac{V_{\text {les }}}{V_{\text {brain }}},
$$

where $V_{\text {brain }}$ is the intracranial brain volume $\left(\mathrm{mm}^{3}\right)$. A meta-analysis recently reported that only $5 \%$ of studies who calculate LL account for intracranial volume [4]. A simple extension of LL is the tractogram load (TL) metric (Fig. 1b), defined as the following ratio:

$$
\mathrm{TL}=\frac{T_{l e s}}{T},
$$

\footnotetext{
${ }^{5}$ www.nottingham.ac.uk/research/groups/clinicalneurology/neuroi.aspx
} 
a) Lesion load

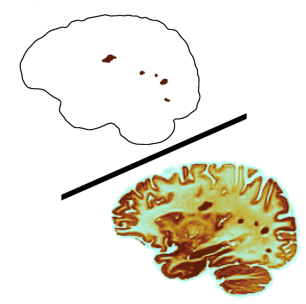

b) Tractogram load

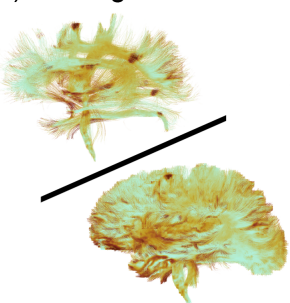

c) Bundle Load

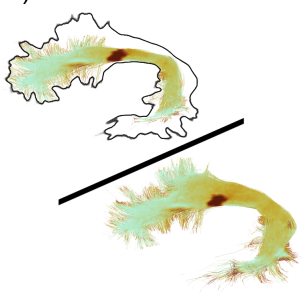

d) Tractometry

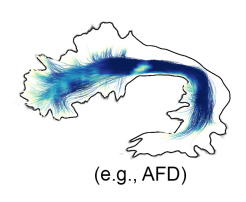

Fig. 1. Graphical overview of various lesion load metrics for an example subject. a) Conventional voxel-based lesion load (normalized by head size). b) Whole-brain tractogram load. c) Topology-based bundle load (example bundle: arcuate fasciculus). d) Lesion-based Tractometry (example bundle: arcuate fasciculus).

where $T_{l e s}$ is the volume of all streamlines (voxelized) passing through all segmented lesions (i.e., the subset of streamlines) and $T$ is the total volume of the whole-brain tractogram. Note that this metric implicitly integrates distal information about the entire streamlines as opposed to local lesion information only $\left(V_{l e s}\right)$. Similarly, the bundle load (BL) metric (Fig. 1c) is a refined sub-case of $\mathrm{TL}$ and can be defined as the following ratio:

$$
\mathrm{BL}=\frac{B_{l e s}}{B},
$$

where, for a given bundle, $B_{\text {les }}$ is the total volume of the subset of streamlines that traverse the lesion (i.e., not to be confounded with $V_{l e s}$ which is the volume of the lesion alone) and $B$ is the total volume of the current bundle-of-interest.

Finally, we also present a lesion-informed Tractometry approach termed here lesionometry (Fig. 1d) defined as:

$$
\text { Lesionometry }=\frac{1}{n m} \sum_{i=1}^{m} \sum_{j=1}^{n} \mathcal{M}\left(s_{i j}\right),
$$

where dMRI measures (e.g., $\mathcal{M}=[\mathrm{FA}, \mathrm{MD}, \mathrm{AFD}, \ldots])$ are sampled at each vertex $j$ forming the streamline $s_{i}$ that is traversing a lesion $\left(B_{l e s}\right)$. If no lesion were present within a bundle, then conventional tract-average was used.

We hypothesize that having a more focused approach around lesions may result in stronger relationships between the metrics and their associated clinical scores. The anticipated directions were as follows: an increase in lesion load (LL, TL, BL) is associated with poorer task performance, and increased AFD is associated with better performance. Pearson correlations were used to assess correlation between the proposed metrics and clinical scores, after correcting for age and gender. Data visualization was done using FiberNavigator [40]. 


\section{$3 \quad$ Results}

\subsection{Lesion mapping}

Fig. 2 (left) shows that most of the lesions were located in deep WM and periventricular areas. Amongst the WM bundles that were extracted, $90 \%$ of the subjects had at least one lesion in the corpus callosum region (Fig 2, right). Fig. 3 qualitatively illustrates the complete reconstruction of the ILF in a single subject where a lesion occurred in the inferior occipital lobe (Fig. 3, green arrow). Streamlines traversing the lesion are color-coded using the anatomical FLAIR image.
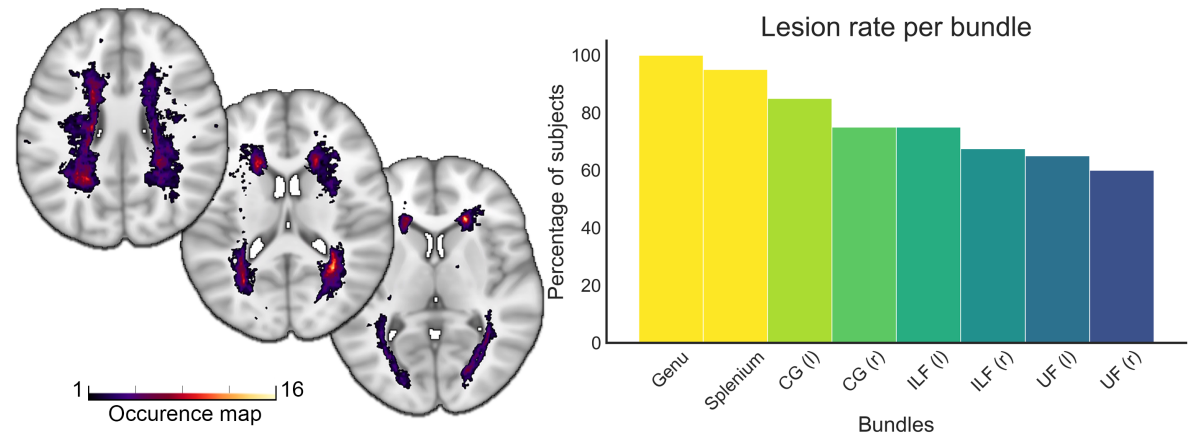

Fig. 2. Density map of all white matter lesions across 40 subjects. The group-average map shows voxels where a lesion was present in at least one of the patients (left). The lesion rate was also derived for the extracted bundles of interests (right). 

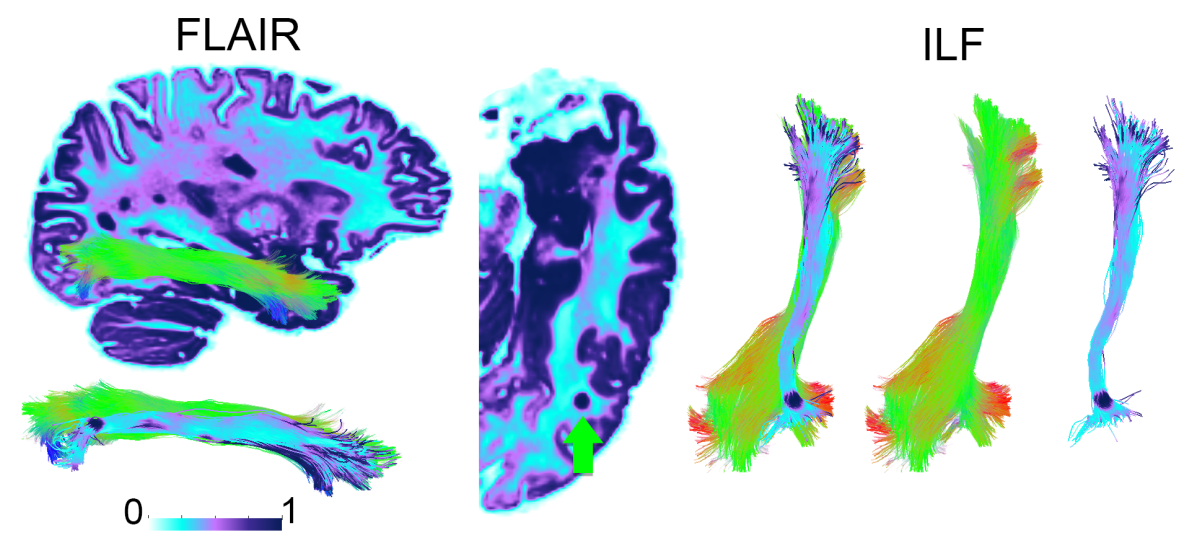

Fig. 3. Tractography of the inferior longitudinal fasciculus (green) in an individual with an MS lesion (green arrow). Streamlines successfully traversing the lesion (right, axial view) are indicated in blue. Color overlay: Intensity normalized FLAIR image.

\subsection{Volumetric metrics}

At the whole-brain level, lesion volume (LV), lesion load (LL) and tractogram load (TL) showed similar negative correlations with MSFC (Fig. 4). Although TL exhibits a slightly lower correlation, the error margin appears less spread than in LV and LL. This could potentially be explained by the presence of outliers in the latter cases. TL also shows that on average, $42 \%$ of the underlying WM architecture can be indirectly affect by lesions. On the other hand, lesion load only affects $0.4 \%$ of the brain.

At the local level, bundle-specific loads (BL) showed stronger associations with tasks than the aforementioned global measures (Fig. 5). For example, the splenium showed stronger association with SoIP (Pearson's $\mathrm{r}=-0.50, \mathrm{p}=0.001$ ) than typical LV (Pearson's $\mathrm{r}=-0.35, \mathrm{p}=0.03$ ). In addition, the bilateral ILF showed greater association with CF-switching (Pearson's $\mathrm{r}=0.35, \mathrm{p}=0.01$ ) than conventional LL (Pearson's $\mathrm{r}=0.30, \mathrm{p}=0.05$ ).

\subsection{Tractometry-based metrics}

Fig. 6 shows results for the lesionometry approach across three tasks. In most cases, the lesion-based tract-averaging (orange) showed greater correlations than typical whole-tract averages (blue). In particular, the link between the left and right CG and LNS (Fig. 6, middle) almost doubled (e.g., from $r=0.18$ to $\mathrm{r}=$ 0.32 and $r=0.17$ to $r=0.31$, respectively). As anticipated, AFD was positively associated with task performance. 

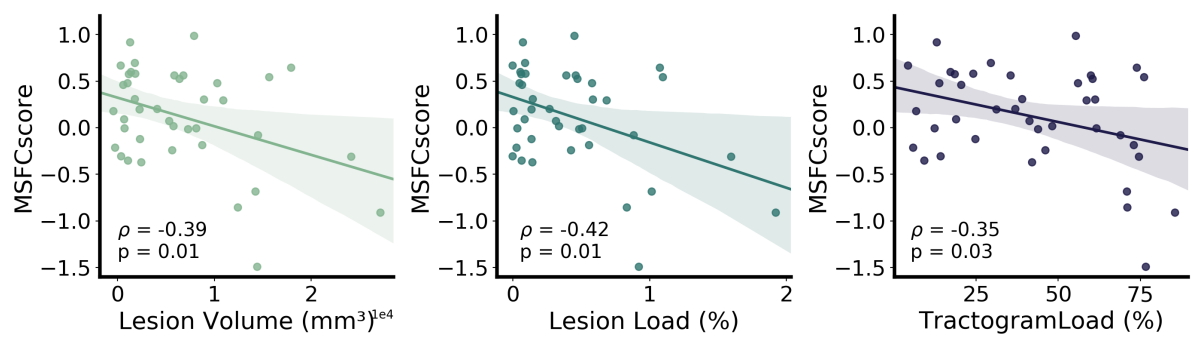

Fig. 4. Global correlations between MSFC and the 3 whole-brain measures (Lesion Volume, Lesion Load and Tractogram Load). As the load increases, the MSFC performance decreases. The correlation between Tracogram Load and MSFC appears to be less driven by outliers.

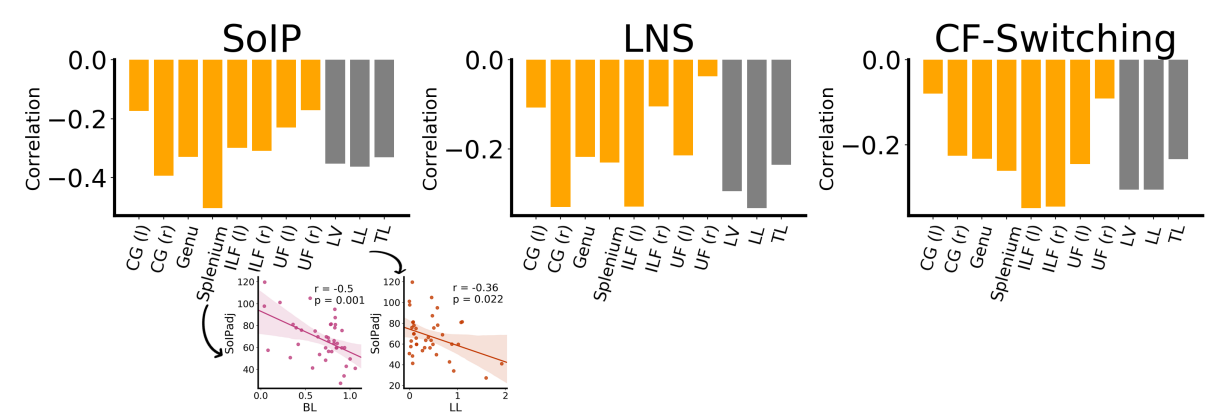

Fig. 5. Bundle load correlations across 3 cognitive tasks show dissociating patterns across bundles. For example, the ILF correlated with CF-switching but not as much with SoIP, whereas the splenium shows correlation with SoIP but not so strongly with LNS.
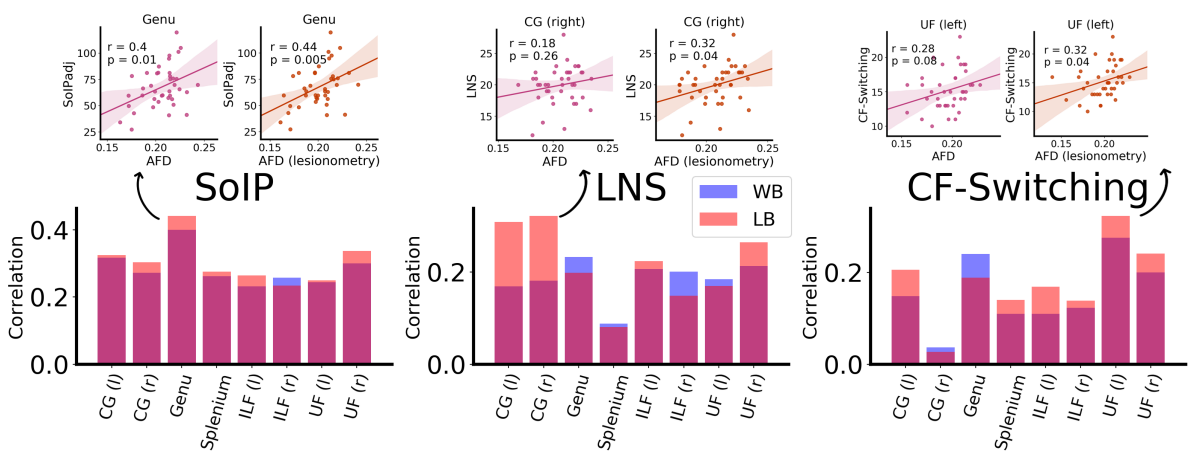

Fig. 6. Lesionometry comparison between whole-bundle (WB, blue) and lesionedbundle (LB, orange) AFD averages. In general, the LB approach shows greater correlations with task than the conventional WB average. The overlapping bar alpha value was set to 0.5 (i.e., red color). 


\section{Discussion and Conclusion}

Focal WM lesions are the hallmark of MS, but inadequately explain disability, creating a clinico-radiological paradox [3,4]. Neuropathology shows that axonal pathology is widespread in the brain in MS. The diffuse axonal damage in MS may be an independent process, or may be driven by anterograde and retrograde degeneration originating at the site of focal lesions [41]. Imaging techniques capable of unravelling the relationship between focal and diffuse pathology, and the correlates of MS disability, remain an unmet need. Diffusion MRI provides quantitative information about WM microstructure. Tractography in MS could provide valuable information about how lesion location relates to key WM bundles, and also inform on the microstructure within lesions and at distant but related sites. However, tractography has been relatively underutilised in MS, perhaps because of technical challenges [16,17]. Here we demonstrate how dMRI and tractography can generate meaningful metrics relating to the burden of lesions, but also their interaction with the structural WM network.

Using lesion mapping, we were able to recreate the known predisposition of WM lesion location [42]. However, we also developed a novel metric by demonstrating the relationship between lesions and WM tracts, which reflected the proportion of the WM tracts that interacted with lesions (TL). Although TL did not improve the strength of correlations beyond LL with a composite measure of disability in people with MS, a reduction in the error margins suggests that this metric may be worthy of further investigation. Nonetheless, it provides a convenient way of visualising the structural network affected by lesions (see Fig. 1b) and also illustrates the high proportion of the WM fibres that interact with a lesion beyond conventional 2D slice-based visualizations.

At the local level, we were able to demonstrate that BL could provide valuable information beyond global metrics to explain task-specific performance. Such a targeted approach may therefore be preferred when trying to relate task performance to specific WM bundles. From a tractometry point-of-view, the lesionometry approach showed stronger association with task performance than typical whole-bundle averages of dMRI measures. Given that focal damage is suspected to extend beyond the visible site of the lesions (i.e., along WM tracts traversing the lesion), sampling dMRI measures selectively within the damaged portion of the bundle may allow more sensitivity to underlying changes in tissue microstructure.

It is already known that the location of the lesions is relevant to clinical disability [43-46]. Furthermore, profiling dMRI measures along WM bundles using the lesionometry approach introduced in this paper, will in theory result in tract profiles that are more sensitive to the underlying lesions. Finally, the metrics introduced in this paper were assessed using an exploratory approach to provide interested readers with an immediate application; undoubtedly, these features could be leveraged in a more advanced context using machine learning. 
In summary, we introduced a set of easy-to-use tract-based metrics to complement existing LL approaches to quantify the extent of brain damage associated with WM lesions in clinical applications.

\section{References}

1. Thompson, A.J., Banwell, B.L., Barkhof, F., Carroll, W.M., Coetzee, T., Comi, G., Correale, J., Fazekas, F., Filippi, M., Freedman, M.S., et al.: Diagnosis of multiple sclerosis: 2017 revisions of the McDonald criteria. The Lancet Neurology 17(2) (2018) 162-173

2. Vellinga, M., Geurts, J., Rostrup, E., Uitdehaag, B., Polman, C., Barkhof, F., Vrenken, H.: Clinical correlations of brain lesion distribution in multiple sclerosis. Journal of Magnetic Resonance Imaging: An Official Journal of the International Society for Magnetic Resonance in Medicine 29(4) (2009) 768-773

3. Barkhof, F.: The clinico-radiological paradox in multiple sclerosis revisited. Curr. Opin. Neurol. 15(3) (Jun 2002) 239-245

4. Mollison, D., Sellar, R., Bastin, M., Mollison, D., Chandran, S., Wardlaw, J., Connick, P.: The clinico-radiological paradox of cognitive function and MRI burden of white matter lesions in people with multiple sclerosis: A systematic review and meta-analysis. PloS One 12(5) (2017)

5. Filippi, M.: Linking structural, metabolic and functional changes in multiple sclerosis. European Journal of Neurology 8(4) (2001) 291-297

6. Mallik, S., Samson, R.S., Wheeler-Kingshott, C.A., Miller, D.H.: Imaging outcomes for trials of remyelination in multiple sclerosis. J Neurol Neurosurg Psychiatry 85(12) (2014) 1396-1404

7. Filippi, M., Preziosa, P., Rocca, M.A.: Microstructural MR imaging techniques in multiple sclerosis. Neuroimaging Clinics 27(2) (2017) 313-333

8. Rocca, M.A., Amato, M.P., De Stefano, N., Enzinger, C., Geurts, J.J., Penner, I.K., Rovira, A., Sumowski, J.F., Valsasina, P., Filippi, M., et al.: Clinical and imaging assessment of cognitive dysfunction in multiple sclerosis. The Lancet Neurology 14(3) (2015) 302-317

9. Stejskal, E.O., Tanner, J.E.: Spin Diffusion Measurements: Spin Echoes in the Presence of a Time-Dependent Field Gradient. The Journal of Chemical Physics 42(1) (jan 1965) 288-292

10. Basser, P., Mattiello, J., Lebihan, D.: Estimation of the Effective Self-Diffusion Tensor from the NMR Spin Echo (1994)

11. Conturo, T.E., Lori, N.F., Cull, T.S., Akbudak, E., Snyder, A.Z., Shimony, J.S., McKinstry, R.C., Burton, H., Raichle, M.E.: Tracking neuronal fiber pathways in the living human brain. Proceedings of the National Academy of Sciences 96(18) (1999) 10422-10427

12. Rovaris, M., Iannucci, G., Falautano, M., Possa, F., Martinelli, V., Comi, G., Filippi, M.: Cognitive dysfunction in patients with mildly disabling relapsingremitting multiple sclerosis: an exploratory study with diffusion tensor MR imaging. Journal of the Neurological Sciences 195(2) (2002) 103-109

13. Rovaris, M., Riccitelli, G., Judica, E., Possa, F., Caputo, D., Ghezzi, A., Bertolotto, A., Capra, R., Falautano, M., Mattioli, F., et al.: Cognitive impairment and structural brain damage in benign multiple sclerosis. Neurology 71(19) (2008) 15211526 
14. Akbar, N., Lobaugh, N.J., O'Connor, P., Moradzadeh, L., Scott, C.J., Feinstein, A.: Diffusion tensor imaging abnormalities in cognitively impaired multiple sclerosis patients. Canadian Journal of Neurological Sciences 37(5) (2010) 608-614

15. Mesaros, S., Rocca, M., Kacar, K., Kostic, J., Copetti, M., Stosic-Opincal, T., Preziosa, P., Sala, S., Riccitelli, G., Horsfield, M., et al.: Diffusion tensor mri tractography and cognitive impairment in multiple sclerosis. Neurology $\mathbf{7 8}(13)$ (2012) 969-975

16. Ciccarelli, O., Catani, M., Johansen-Berg, H., Clark, C., Thompson, A.: Diffusionbased tractography in neurological disorders: concepts, applications, and future developments. The Lancet Neurology 7(8) (2008) 715-727

17. Lipp, I., Parker, G.D., Tallantyre, E.C., Goodall, A., Grama, S., Patitucci, E., Heveron, P., Tomassini, V., Jones, D.K.: Tractography in the presence of multiple sclerosis lesions. NeuroImage 209 (Apr 2020) 116471

18. Jeurissen, B., Tournier, J.D., Dhollander, T., Connelly, A., Sijbers, J.: Multi-tissue constrained spherical deconvolution for improved analysis of multi-shell diffusion MRI data. NeuroImage 103 (2014) 411-426

19. Smith, R.E., Tournier, J.D., Calamante, F., Connelly, A.: Anatomicallyconstrained tractography: improved diffusion mri streamlines tractography through effective use of anatomical information. NeuroImage 62(3) (2012) 1924-1938

20. Girard, G., Whittingstall, K., Deriche, R., Descoteaux, M.: Towards quantitative connectivity analysis: reducing tractography biases. NeuroImage 98 (2014) 266278

21. Gajamange, S., Raffelt, D., Dhollander, T., Lui, E., van der Walt, A., Kilpatrick, T., Fielding, J., Connelly, A., Kolbe, S.: Fibre-specific white matter changes in multiple sclerosis patients with optic neuritis. NeuroImage: Clinical 17 (2018) 60-68

22. Mito, R., Raffelt, D., Dhollander, T., Vaughan, D.N., Tournier, J.D., Salvado, O., Brodtmann, A., Rowe, C.C., Villemagne, V.L., Connelly, A.: Fibre-specific white matter reductions in alzheimer's disease and mild cognitive impairment. Brain 141(3) (2018) 888-902

23. Tur, C., Grussu, F., Prados, F., Charalambous, T., Collorone, S., Kanber, B., Cawley, N., Altmann, D.R., Ourselin, S., Barkhof, F., et al.: A multi-shell multitissue diffusion study of brain connectivity in early multiple sclerosis. Multiple Sclerosis Journal (2019) 1352458519845105

24. Charalambous, T., Tur, C., Prados, F., Kanber, B., Chard, D.T., Ourselin, S., Clayden, J.D., Wheeler-Kingshott, C.A.G., Thompson, A.J., Toosy, A.T.: Structural network disruption markers explain disability in multiple sclerosis. Journal of Neurology, Neurosurgery \& Psychiatry 90(2) (2019) 219-226

25. Dumont, M., Roy, M., Jodoin, P.M., Morency, F.C., Houde, J.C., Xie, Z., Bauer, C., Samad, T.A., Van Dijk, K.R., Goodman, J., et al.: Free water in white matter differentiates mci and ad from control subjects. Frontiers in Aging Neuroscience 11 (2019) 270

26. Beaudoin, A.M., Rheault, F., Theaud, G., Whittingstall, K., Lamontagne, A., Descoteaux, M.: White matter tractometry correlates with fatigue severity in young adults with multiple sclerosis (1322). Neurology 94(15 Supplement) (2020)

27. Storelli, L., Pagani, E., Preziosa, P., Filippi, M., Rocca, M.A.: Measurement of white matter fiber-bundle cross-section in multiple sclerosis using diffusionweighted imaging. Multiple Sclerosis Journal (2020) 1352458520938999

28. Maier-Hein, K.H., Neher, P.F., Houde, J.C., Côté, M.A., Garyfallidis, E., Zhong, J., Chamberland, M., Yeh, F.C., Lin, Y.C., Ji, Q., Others: The challenge of mapping 
the human connectome based on diffusion tractography. Nature Communications 8 (2017) 1349

29. Jones, D.K., Knösche, T.R., Turner, R.: White matter integrity, fiber count, and other fallacies: The do's and don'ts of diffusion MRI. NeuroImage 73 (2013) 239254

30. Schilling, K.G., Nath, V., Hansen, C., Parvathaneni, P., Blaber, J., Gao, Y., Neher, P., Aydogan, D.B., Shi, Y., Ocampo-Pineda, M., et al.: Limits to anatomical accuracy of diffusion tractography using modern approaches. NeuroImage 185 (2019) 1-11

31. Wasserthal, J., Neher, P., Maier-Hein, K.H.: TractSeg - Fast and accurate white matter tract segmentation. NeuroImage 183 (dec 2018) 239-253

32. Tallantyre, E.C., Major, P.C., Atherton, M.J., Davies, W.A., Joseph, F., Tomassini, V., Pickersgill, T.P., Harding, K.E., Willis, M.D., Winter, M., et al.: How common is truly benign $\mathrm{ms}$ in a uk population? Journal of Neurology, Neurosurgery \& Psychiatry 90(5) (2019) 522-528

33. Fischer, J., Rudick, R., Cutter, G., Reingold, S., Force, N.M.S.C.O.A.T.: The multiple sclerosis functional composite measure (MSFC): an integrated approach to ms clinical outcome assessment. Multiple Sclerosis Journal 5(4) (1999) 244-250

34. Weiss, L.G., Saklofske, D.H., Coalson, D., Raiford, S.E.: WAIS-IV clinical use and interpretation: Scientist-practitioner perspectives. Academic Press (2010)

35. Coughlan, A., Oddy, M., Crawford, J.: Birt memory and information processing battery (bmipb). London: Brain Injury Rehabilitation Trust (2007)

36. Delis, D.C., Kaplan, E., Kramer, J.H.: Delis-kaplan executive function system. (2001)

37. Veraart, J., Novikov, D.S., Christiaens, D., Ades-Aron, B., Sijbers, J., Fieremans, E.: Denoising of diffusion MRI using random matrix theory. NeuroImage 142 (2016) 394-406

38. Andersson, J.L.R., Sotiropoulos, S.N.: An integrated approach to correction for off-resonance effects and subject movement in diffusion MR imaging. NeuroImage 125 (2016) 1063-1078

39. Andersson, J.L.R., Skare, S., Ashburner, J.: How to correct susceptibility distortions in spin-echo echo-planar images: application to diffusion tensor imaging. NeuroImage 20(2) (2003) 870-888

40. Chamberland, M., Whittingstall, K., Fortin, D., Mathieu, D., Descoteaux, M.: Real-time multi-peak tractography for instantaneous connectivity display. Frontiers in Neuroinformatics 8 (jan 2014) 59

41. Filippi, M., Brück, W., Chard, D., Fazekas, F., Geurts, J.J., Enzinger, C., Hametner, S., Kuhlmann, T., Preziosa, P., Rovira, À., et al.: Association between pathological and mri findings in multiple sclerosis. The Lancet Neurology 18(2) (2019) $198-210$

42. Holland, C.M., Charil, A., Csapo, I., Liptak, Z., Ichise, M., Khoury, S.J., Bakshi, R., Weiner, H.L., Guttmann, C.R.: The relationship between normal cerebral perfusion patterns and white matter lesion distribution in 1,249 patients with multiple sclerosis. Journal of Neuroimaging 22(2) (2012) 129-136

43. Filippi, M., Rocca, M.A., Martino, G., Horsfield, M.A., Comi, G.: Magnetization transfer changes in the normal appering white matter precede the appearance of enhancing lesions in patients with multiple sclerosis. Annals of neurology 43(6) (1998) 809-814

44. Thiebaut de Schotten, M., Dell'Acqua, F., Ratiu, P., Leslie, A., Howells, H., Cabanis, E., Iba-Zizen, M., Plaisant, O., Simmons, A., Dronkers, N., et al.: From 
phineas gage and monsieur leborgne to hm: revisiting disconnection syndromes. Cerebral Cortex 25(12) (2015) 4812-4827

45. Foulon, C., Cerliani, L., Kinkingnehun, S., Levy, R., Rosso, C., Urbanski, M., Volle, E., Thiebaut de Schotten, M.: Advanced lesion symptom mapping analyses and implementation as bcbtoolkit. Gigascience 7(3) (2018) giy004

46. Fox, M.D.: Mapping symptoms to brain networks with the human connectome. New England Journal of Medicine 379(23) (2018) 2237-2245 